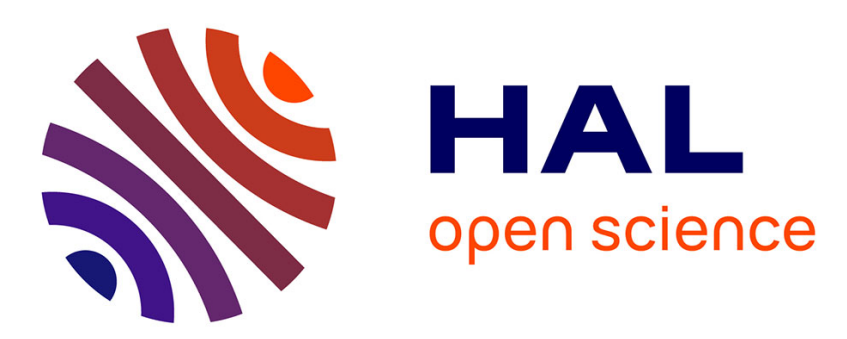

\title{
MECANISME DES REACTIONS (t, d) SUR 6Li, 9Be, ET 10B POUR DES TRITONS D'ENERGIE INFERIEURE A 3,5 MeV
}

\author{
O. Bing, R. Seltz, C. Gerardin, M. Wery
}

\section{- To cite this version:}

O. Bing, R. Seltz, C. Gerardin, M. Wery. MECANISME DES REACTIONS (t, d) SUR 6Li, 9Be, ET 10B POUR DES TRITONS D'ENERGIE INFERIEURE A 3,5 MeV. Journal de Physique Colloques, 1970, 31 (C2), pp.C2-142-C2-143. 10.1051/jphyscol:1970241 . jpa-00213799

HAL Id: jpa-00213799

https://hal.science/jpa-00213799

Submitted on 1 Jan 1970

HAL is a multi-disciplinary open access archive for the deposit and dissemination of scientific research documents, whether they are published or not. The documents may come from teaching and research institutions in France or abroad, or from public or private research centers.
L'archive ouverte pluridisciplinaire HAL, est destinée au dépôt et à la diffusion de documents scientifiques de niveau recherche, publiés ou non, émanant des établissements d'enseignement et de recherche français ou étrangers, des laboratoires publics ou privés. 
MECANISME DES RERCTIONS ( $t, d$ ) SUR ${ }^{6}$ Li, ${ }^{9}$ Be ET ${ }^{10}$ B POUR des TRITONS d'ENERGIE INFERIEURE A $3,5 \mathrm{MEV}$

O.BING, R.SELTZ, C.GERARDIN, M.WERY

Laboratoire des Basses Energies - CRN - (67) STRASBOURG 3

\begin{abstract}
Résumé - Nous avons mesuré les courbes d'excitation et les distributions angulaires des réactions $(t, d)$ sur $6 L i, 9_{B e}$ et $10 \mathrm{~B}$ pour des énergies incidentes comprises entre 1,5 et $3,5 \mathrm{MeV}$. Le calcul en D.W.B.A. pour un mécanisme de stripping donne un bon accord qualitatif sur les distributions angulaires mesurées dans le cas des réactions $6_{L i(t, d)}{ }^{7}$ Li et $9_{B e}(t, d) 10 B e$. Pour la réaction $10_{B}(t, d) 11_{B} l$ laccord n'est que partiel et montre la contribution d'autres mécanismes directs.
\end{abstract}

\begin{abstract}
Differential cross section have been measured between 1.5 and $3.5 \mathrm{MeV}$ for the ${ }^{6} \mathrm{Li}(t, d)^{7} \mathrm{Li},{ }_{\mathrm{Be}}(t, d){ }^{10} \mathrm{Be}$ and $10_{B}(t, d)^{11} \mathrm{~B}$ reactions going to the ground states of residual nuclei. D.W.B.A. calculations provide satisfactory fits to the. $\mathrm{CL}_{\mathrm{L}} \mathrm{i}$ and $9_{\mathrm{Be}}$ reactions. For the $10_{\mathrm{B}}(\mathrm{t}, \mathrm{d}) 1_{\mathrm{B}}$ reaction a single stripping mechanism is unable to explain the large backward peak.
\end{abstract}

On s'attend ̀̀ ce que les réactions (t,d) aient une grande similitude avec les rébetions $(d, p)$ reliant les mêmes états. Aux énergies inférieures a 2 MeV, et pour la réaction ${ }^{14} \mathrm{~N}(t, d){ }^{15} \mathrm{~N}$, une interprëtation en mécanisme de strip-

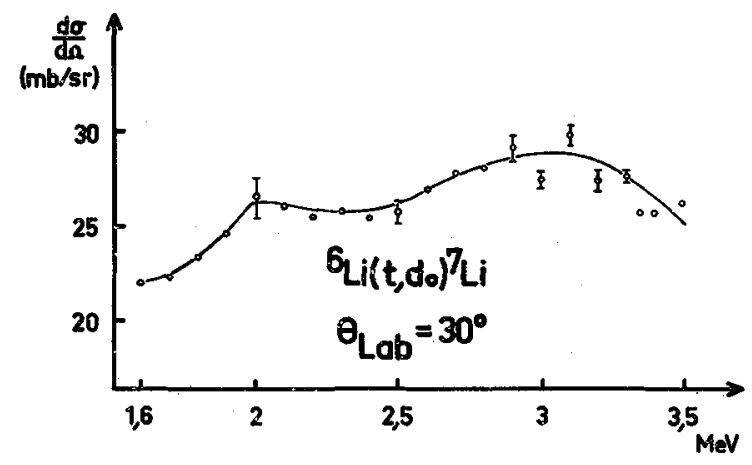

ping s'est avérée incapable de reproduire les mesures aussi bien qualitativement que quartitativement [1]. Il nous a paru intéressant d'étudier les réactions $(t, d)$ sur d'autres noyaux de la couche $1 p$ [2]. Pour les trois réactions choisies, les courbes d'excitation sont monotones dans la région d'excitation étudiée (fig. 1, 2 ). Les calculs en D.W.B.A. avec l'hypothèse d'un mecanisme de stripping ont été effectués avec les paramètres résumes dans le tableau I. Ces paramètres ont été en partie déterminés par nous, en partie tirés de la littérature.

GLi(t,do) ${ }^{7} L i$ [fig. 3 : L'accord qualitatif est satisfaisant sur l'ensemble de la distribution angulaire, mais la position du premier maximum est mal reproduite,

9Be(t,d)10 $10_{B e}$ (fig. 4) : L'accord est satisfaisant et confirme ce qui est attendu à partir des résultats de la réaction

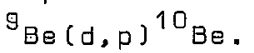

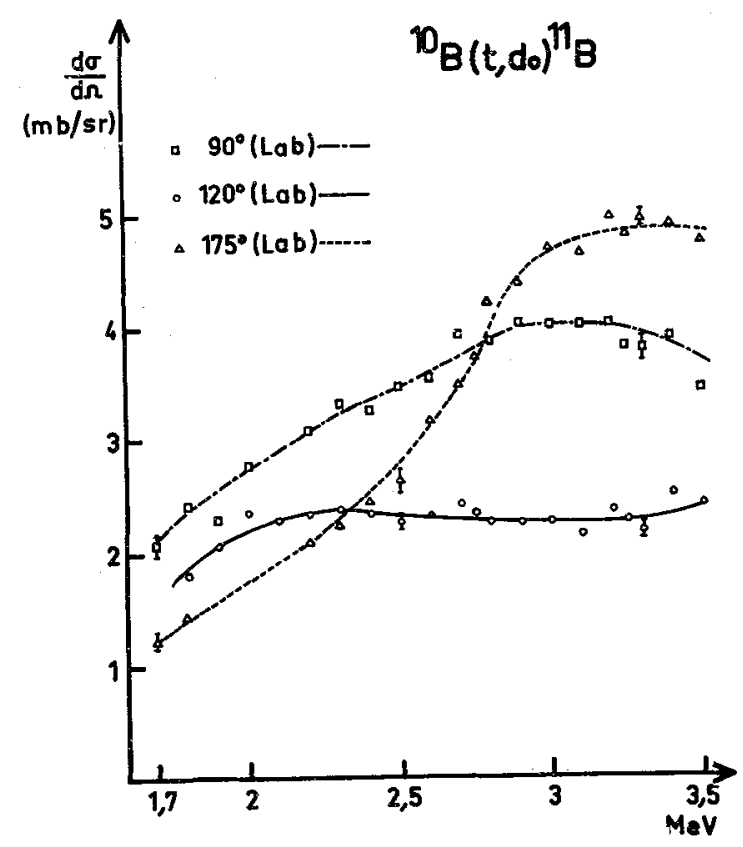




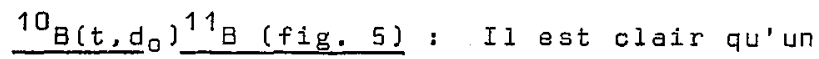
simple mécanisme de stripping est incapable de rendre compte des trois maximums observés dans la distribution angulaire. Remarquons cependant que le moment de transfert $\ell_{p}=3$ semble plus approprié sur l'ensemble du domaine étudié. En effet, les deux maximums à l'avant et à l'arrière n'apparaissent qu'au-dessus de $2,5 \mathrm{MeV}$ d'énergie incidente.

BIBLIOGRAPHIE

[1] R.8.SCHWARTZ et al

Phys. Rev. 134 (1964) B 577

[2] 0.BING. Thèse de 3e cycle strasbourg.
B

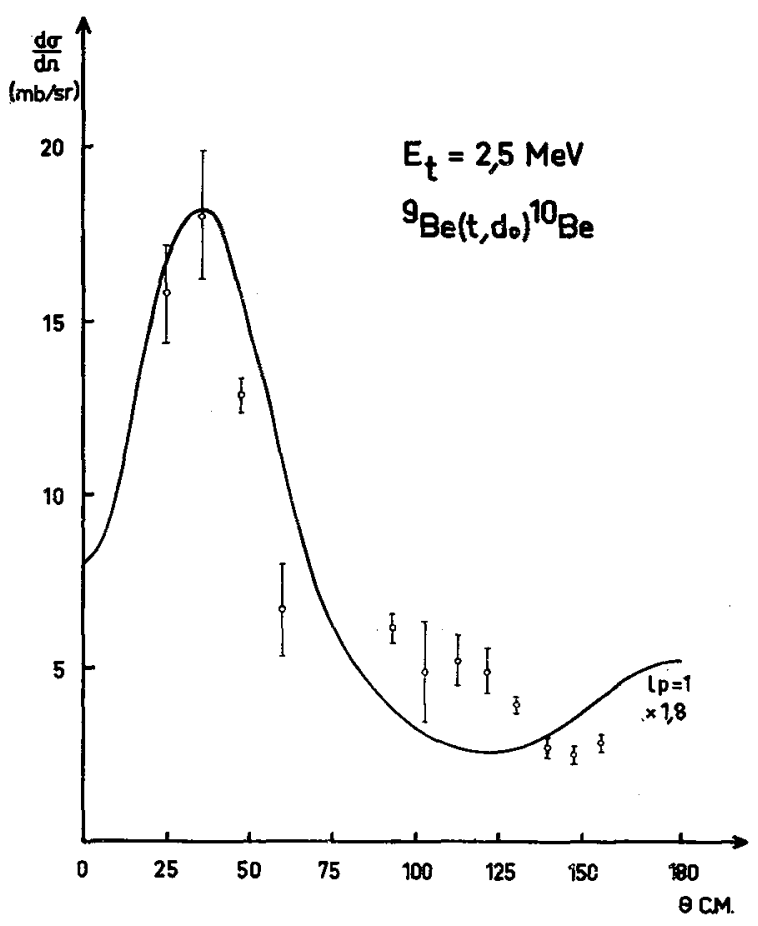

FIGURE 3

\begin{tabular}{|c|c|c|c|c|c|c|c|c|}
\hline \multirow{3}{*}{$\begin{array}{l}\text { Réaction } \\
10_{B(t, d)^{11} B}\end{array}$} & Voie & $u$ & $W$ & $r_{r}$ & ${ }_{r}$ & $r_{i}$ & $a_{i}$ & roc \\
\hline & Entrée & 122 & 0,95 & 0,85 & 0,704 & 1.23 & 0.959 & 1,39 \\
\hline & Sortie & 118 & 4,82 & 0.895 & 0,902 & 1,62 & 0.775 & 1,3 \\
\hline \multirow[t]{2}{*}{$\mathrm{B} e(t, d)^{10} \mathrm{Be}$} & Entrée & 1.45 & 1,91 & 0,85 & 0,704 & 2,06 & 0,772 & 1,3 \\
\hline & Sortie & 40,34 & 6,58 & 1,48 & 0.63 & 0,48 & 0,63 & 1,3 \\
\hline \multirow[t]{2}{*}{${ }^{6} L i(t, d)^{7} L i$} & Entrée & 38,89 & 8,66 & 1,65 & 0,5 & 1,65 & 0.5 & 1,65 \\
\hline & Sortie & 34,9 & 5.77 & 1,5 & 0,59 & 1,5 & 0.59 & 1,3 \\
\hline
\end{tabular}

TABLEAU I

Les potentiels sont en MeV et les rayons en fermi

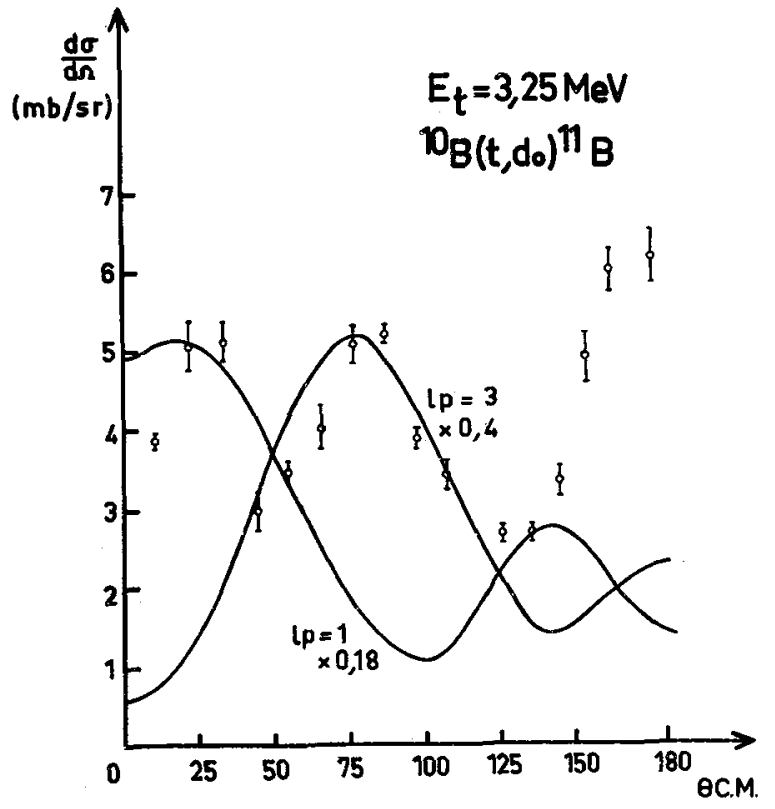

FIGURE 4

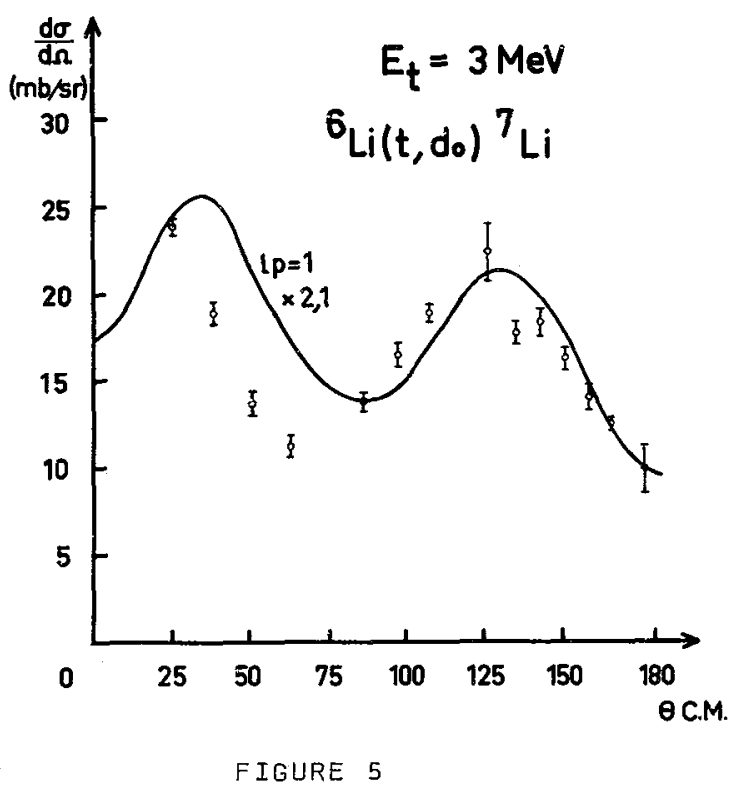

